\title{
An exact transverse Helmholtz equation
}

\author{
Bruno Crosignani, ${ }^{1,3, *}$ Paolo Di Porto, ${ }^{2}$ and Amnon Yariv ${ }^{3}$ \\ ${ }^{1}$ Dipartimento di Fisica, Universita' dell'Aquila, 67100 L'Aquila, Italy, and Centro di Ricerca e Sviluppo SOFT.CNR, \\ Universita' di Roma "La Sapienza," 00185 Roma, Italy \\ ${ }^{2}$ Dipartimento di Fisica, Universita' dell'Aquila, 67100 L'Aquila, Italy, and Laboratorio Regionale CASTI-CNR, \\ Universita' dell'Aquila, 67100 L'Aquila, Italy \\ ${ }^{3}$ Department of Applied Physics, California Institute of Technology, Pasadena, California 91125, USA \\ *Corresponding author: bcross@caltech.edu
}

Received June 26, 2009; accepted July 7, 2009;

posted July 17, 2009 (Doc. ID 113362); published August 19, 2009

We derive an exact equation for the transverse component of the electric field propagating along a given longitudinal $\mathrm{z}$ direction in the presence of an isotropic refractive-index distribution $\mathrm{n}(\mathrm{x}, \mathrm{y})$. (C) 2009 Optical Society of America

OCIS codes: $260.0260,350.7420,350.5500$.

\section{INTRODUCTION}

Monochromatic wave propagation in an isotropic lossless material medium in the presence of a refractive index distribution $\mathrm{n}(\mathrm{x}, \mathrm{y}, \mathrm{z})$ is most often dealt with by relying on the so-called Helmholtz equation [1]. It can be derived in a straightforward way from the macroscopic Maxwell equations under the assumption that the so-called polarization-mixing term $\nabla \nabla \cdot \mathbf{E}$ is zero, this being in general not the case unless the refractive index is a constant. The resulting scalar equation, the Helmholtz equation, is intrinsically limited by the assumption $\nabla \nabla \cdot \mathbf{E}=0$. Keeping this in mind, one looks for the solution of the Helmholtz equation containing only the transverse $(\mathrm{x}, \mathrm{y})$ components of the field $\mathbf{E}_{\perp}$ and successively derives its longitudinal component $\mathrm{E}_{\mathrm{z}}$ by taking advantage of the divergence condition $\nabla \cdot\left(\mathrm{n}^{2} \mathbf{E}\right)=0$. In many cases, it turns out to be convenient to resort to a further approximation, the paraxial one, which basically restricts the scale of variation of the amplitude of the field propagating in the $\mathrm{z}$ direction to values much larger than the wavelength (slowly-varying approximation, SVA). The main limitations of the resulting paraxial equation are associated with its scalar character (inherited from the Helmholtz equation), which does not allow for the presence of polarization coupling nor for a complete description of diffraction effects. A few attempts have been made to improve the above scenario, mainly aimed at including polarization effects and nonparaxial diffraction contributions higher than the first in a smallnesss parameter $\varepsilon=\lambda / \ell$ (where $\ell$ is the beam waist); the most recent one is limited to terms of order $\varepsilon^{2}$ [2].

The main contribution of this paper consists of the derivation, for a z-independent isotropic refractive-index distribution $\mathrm{n}(\mathrm{x}, \mathrm{y})$ (the case in typical optical waveguides), of an exact vectorial wave equation for the transverse component of the electric field. By relying on it, one can also derive, by adopting the SVA, an improved version of the standard paraxial equation.

\section{HELMHOLTZ EQUATION AND PARAXIAL EQUATION}

The description of propagation of a monochromatic electric field in a medium described by an isotropic refractiveindex $\mathrm{n}(\mathbf{r})=\mathrm{n}_{0}+\delta \mathrm{n}(\mathbf{r})$ is completely vectorial in nature and reads [1]

$$
\nabla^{2} \mathbf{E}-\nabla \nabla \cdot \mathbf{E}+\frac{\omega^{2}}{\mathrm{c}^{2}} \mathrm{n}^{2}(\mathbf{r}) \mathbf{E}=0
$$

where $\nabla^{2}=\nabla_{\perp}^{2}+\partial^{2} / \partial \mathrm{z}^{2}$ and $\nabla_{\perp}^{2}=\partial^{2} / \partial \mathrm{x}^{2}+\partial^{2} / \partial \mathrm{y}^{2}$.

In particular, if it is a priori known (or approximately assumed) that

$$
\nabla \nabla \cdot \mathbf{E}=0
$$

Eq. (1) takes the form of the standard Helmholtz equation, that is,

$$
\nabla^{2} \mathbf{E}+\frac{\omega^{2}}{\mathrm{c}^{2}} \mathrm{n}^{2}(\mathbf{r}) \mathbf{E}=0 .
$$

In general, Eq. (2) does not apply and Eq. (3) is an approximate one. To solve it, a well-known approach is to rely on the paraxial approximation, that is, to consider the field as completely transverse and to neglect the term containing the second derivative with respect to $\mathrm{z}$.

In the standard paraxial approach, the field $\mathbf{E}=\mathbf{E}_{\perp}$ $+\mathbf{E}_{\mathrm{z}}$ is assumed to be approximately transverse to the propagation direction $\mathrm{z}\left(\left|\mathbf{E}_{\mathrm{z}} / \mathbf{E}_{\perp}\right| \ll 1\right)$ and written in the form (with $\mathrm{k}=\mathrm{n}_{0} \omega / \mathrm{c}$ )

$$
\mathbf{E}_{\perp}(\mathbf{r})=\mathbf{A}(\mathbf{r}) \exp (\mathrm{ikz}) .
$$

After assuming Eq. (3) to be valid, Eq. (4) is inserted into it and the $\partial_{\mathrm{zz}}^{2} \mathbf{A}$ term is neglected. This last simplification is valid if one assumes-in the frame of the SVA$\mathbf{A}\left(\mathbf{r}_{\perp}, \mathrm{z}\right)$ to vary slowly, as a function of $\mathrm{z}$, on a scale of the order of $1 / \mathrm{k}$. If we now define $\Delta \mathrm{n}=\left(\mathrm{n}^{2}-\mathrm{n}_{0}^{2}\right) / 2 \mathrm{n}_{0}^{2}$, the resulting equation reads 


$$
2 \mathrm{ik} \partial_{\mathrm{z}} \mathbf{A}+\nabla_{\perp}^{2} \mathbf{A}+2 \mathrm{k}^{2} \Delta \mathrm{n} \mathbf{A}=0
$$

which is usually referred to as the paraxial equation.

One of the main limitations of the above approach (besides its scalar nature preventing polarization-coupling and bidirectional propagation) is that it is able to account for diffraction only to the lowest significant order in the smallness parameter $\varepsilon^{2}=\nabla_{\perp}^{2} / \mathrm{k}^{2} \approx(1 / \ell \mathrm{k})^{2}$, where $\ell$ is a typical transverse scale of the beam, the higher-order terms having been lost as a consequence of the omission of the term in $\partial^{2} / \partial z^{2}$.

\section{EXACT APPROACH}

Is it possible, starting from Maxwell's equations, to develop a theory describing the propagation of the transverse component of the field without explicitly requiring that $\nabla \nabla \cdot \mathbf{E}=0$ ? This has been shown [3] to be possible by means of an alternative description of propagation based on the introduction of two auxiliary transverse fields $\mathbf{E}^{\prime}$ and $\mathbf{E}^{\prime \prime}$ obeying a coupled system of two first-order (in $\partial_{\mathrm{z}}$ ) equations and whose sum $\mathbf{E}^{\prime}+\mathbf{E}^{\prime \prime}$ reproduces the transverse component $\mathbf{E}_{\perp}$ of $\mathbf{E}$. In particular, the boundary conditions for the field and its derivative at $z=0$ required for solving Eq. (1) are replaced by the knowledge of the values of $\mathbf{E}^{\prime}$ and $\mathbf{E}^{\prime \prime}$ at $\mathrm{z}=0$.

More precisely, the transverse component of the electric field is expressed in the form

$$
\begin{aligned}
\mathbf{E}_{\perp}\left(\mathbf{r}_{\perp}, \mathrm{z}\right)= & \mathbf{E}^{\prime}+\mathbf{E}^{\prime \prime} \\
= & \int \mathrm{d} \mathbf{k}_{\perp} \exp \left(\mathrm{i} \mathbf{k}_{\perp} \cdot \mathbf{r}_{\perp}\right){\boldsymbol{\varepsilon}^{\prime}}_{\perp}\left(\mathbf{k}_{\perp}, \mathrm{z}\right) \exp \left[\mathrm{ih}\left(\mathrm{k}_{\perp}\right) \mathrm{z}\right] \\
& +\boldsymbol{\varepsilon}^{\prime \prime}{ }_{\perp}\left(\mathbf{k}_{\perp}, \mathrm{z}\right) \exp \left[-\mathrm{ih}\left(\mathrm{k}_{\perp}\right) \mathrm{z}\right],
\end{aligned}
$$

where $\mathrm{h}\left(\mathrm{k}_{\perp}\right)=\left(\mathrm{k}^{2}-\mathrm{k}_{\perp}^{2}\right)^{1 / 2}$, an analogous expression holding for the magnetic field $\mathbf{B}_{\perp}$. Note that if $\boldsymbol{\varepsilon}^{\prime}{ }_{\perp}\left(\mathbf{k}_{\perp}, \mathbf{z}\right)$ and $\boldsymbol{\varepsilon}^{\prime \prime}{ }_{\perp}\left(\mathbf{k}_{\perp}, \mathbf{z}\right)$ were strongly peaked functions of $\mathbf{k}_{\perp}, \mathbf{E}^{\prime}$ and $\mathbf{E}^{\prime \prime}$ could be identified, as a first approximation, with the forward and backward traveling field components.

By introducing $\mathbf{E}_{\perp}$ and $\mathbf{B}_{\perp}$ in the corresponding Maxwell's equations for the transverse components and introducing the differential operator $\hat{\mathrm{L}}=\left(\mathrm{k}^{2}+\nabla_{\perp}^{2}\right)^{1 / 2}$, one can derive the following system of coupled equations for $\mathbf{E}^{\prime}$ and $\mathbf{E}^{\prime \prime}$ [see [3], Eq. (E1). In particular, for an isotropic refractive-index distribution, it reads

$$
\begin{aligned}
\left(\mathrm{i} \frac{\partial}{\partial \mathrm{z}}+\hat{\mathrm{L}}\right) \mathbf{E}^{\prime}= & -\frac{\mathrm{k}^{2}}{\hat{\mathrm{L}}}\left(\Delta \mathrm{n} \mathbf{E}_{\perp}\right)+\nabla_{\perp}\left[\Delta \mathrm{n} \frac{1}{\hat{\mathrm{L}}} \nabla_{\perp} \cdot\left(\mathbf{E}^{\prime}-\mathbf{E}^{\prime \prime}\right)\right] \\
& -\frac{1}{\hat{\mathrm{L}}} \nabla_{\perp}\left[\nabla_{\perp} \cdot\left(\Delta \mathrm{n} \mathbf{E}_{\perp}\right)\right] \\
\left(\mathrm{i} \frac{\partial}{\partial \mathrm{z}}-\hat{\mathrm{L}}\right) \mathbf{E}^{\prime \prime}= & \frac{\mathrm{k}^{2}}{\hat{\mathrm{L}}}\left(\Delta \mathrm{n} \mathbf{E}_{\perp}\right)+\nabla_{\perp}\left[\Delta \mathrm{n} \frac{1}{\hat{\mathrm{L}}} \nabla_{\perp} \cdot\left(\mathbf{E}^{\prime}-\mathbf{E}^{\prime \prime}\right)\right] \\
& +\frac{1}{\hat{\mathrm{L}}} \nabla_{\perp}\left[\nabla_{\perp} \cdot\left(\Delta \mathrm{n} \mathbf{E}_{\perp}\right)\right] .
\end{aligned}
$$

Solving the above set of equations is completely equiva- lent to solving Eq. (1). The main advantage of using the set of Eqs. (7) is the first-order dependence on $\partial_{z}$, the expansion of the differential operators $\hat{\mathrm{L}}$ to any desired degree of approximation in the smallness parameter $\varepsilon^{2}$ $=\nabla_{\perp}^{2} / \mathrm{k}^{2} \cong(1 / \mathrm{k} \ell)^{2}$ allowing for the inclusion of all-order nonparaxial contributions.

One can now derive, whenever $\Delta \mathrm{n}(\mathrm{x}, \mathrm{y}, \mathrm{z})=\Delta \mathrm{n}(\mathrm{x}, \mathrm{y})$, and starting from the set of Eqs. (7) describing (2+1)-D propagation, a single equation for the transverse part of the field $\mathbf{E}_{\perp}=\mathbf{E}^{\prime}+\mathbf{E}^{\prime \prime}$. This equation will represent, without approximations, the rigorous version of the Helmholtz equation for the transverse component of the field. In particular, if advantage is taken of the SVA hypothesis, this will produce an equation that is the generalization of the standard paraxial equation.

In order to prove the above assertion, we add and subtract Eq. (7a) and Eq. (7b), thus getting an equivalent set of coupled equations for the variables $\mathbf{E}^{\prime}+\mathbf{E}^{\prime \prime}=\mathbf{E}_{\perp}$ and $\mathbf{E}^{\prime}-\mathbf{E}^{\prime \prime}$ that read

$$
\begin{aligned}
\mathrm{i} \frac{\partial}{\partial \mathrm{z}} \mathbf{E}_{\perp}+\hat{\mathrm{L}}\left(\mathbf{E}^{\prime}-\mathbf{E}^{\prime \prime}\right)= & 2 \nabla_{\perp}\left[\Delta \mathrm{n} \frac{1}{\hat{\mathrm{L}}} \nabla_{\perp} \cdot\left(\mathbf{E}^{\prime}-\mathbf{E}^{\prime \prime}\right)\right], \\
\mathrm{i} \frac{\partial}{\partial \mathrm{z}}\left(\mathbf{E}^{\prime}-\mathbf{E}^{\prime \prime}\right)+\hat{\mathrm{L}} \mathbf{E}_{\perp} & =-2 \frac{\mathrm{k}^{2}}{\hat{\mathrm{L}}}\left(\Delta \mathrm{n} \mathbf{E}_{\perp}\right) \\
& -\frac{2}{\hat{\mathrm{L}}} \nabla_{\perp}\left[\nabla_{\perp} \cdot\left(\Delta \mathrm{n} \mathbf{E}_{\perp}\right)\right] .
\end{aligned}
$$

Applying $\partial / \partial z$ on both sides of Eq. (8a) and taking advantage of Eq. (8b) yields, after some algebra, the following equation for $\mathbf{E}_{\perp}$ :

$$
\begin{aligned}
& \frac{\partial^{2}}{\partial \mathrm{z}^{2}} \mathbf{E}_{\perp}+\nabla_{\perp}^{2} \mathbf{E}_{\perp}+\mathrm{k}^{2} \mathbf{E}_{\perp}+2 \mathrm{k}^{2}\left(\Delta \mathrm{n} \mathbf{E}_{\perp}\right)+2 \nabla_{\perp}\left(\mathbf{E}_{\perp} \cdot \nabla_{\perp} \Delta \mathrm{n}\right) \\
& \quad=4 \nabla_{\perp}\left\{\Delta \mathrm{n}\left[\mathrm{k}^{2}+\nabla_{\perp} \cdot \nabla_{\perp}\right]\left[\frac{1}{\hat{\mathrm{L}}^{2}} \nabla_{\perp} \cdot\left(\Delta \mathrm{n} \mathbf{E}_{\perp}\right)\right]\right\} \\
& \quad=4 \nabla_{\perp}\left[\Delta \mathrm{n} \nabla_{\perp} \cdot\left(\Delta \mathrm{n} \mathbf{E}_{\perp}\right)\right],
\end{aligned}
$$

which can be rewritten in the final compact form

$$
\begin{aligned}
& \nabla^{2} \mathbf{E}_{\perp}+\mathrm{k}^{2}\left(\mathrm{n} / \mathrm{n}_{0}\right)^{2} \mathbf{E}_{\perp}+2 \nabla_{\perp}\left\{\mathbf{E}_{\perp} \cdot \nabla_{\perp}\left[\Delta \mathrm{n}+(\Delta \mathrm{n})^{2}\right]\right\} \\
& \quad=4 \nabla_{\perp}\left[(\Delta \mathrm{n})^{2} \nabla_{\perp} \cdot \mathbf{E}_{\perp}\right] .
\end{aligned}
$$

Equation (10), fully transverse and including all diffraction and polarization-coupling contributions, is the exact transverse Helmholtz equation, which constitutes the main result of this paper. It is worth noting the presence of the term $(\Delta n)^{2}$, which is typically a small number in conventional optical waveguides $\left(\Delta \mathrm{n} \cong 10^{-2}-10^{-3}\right)$. If terms quadratic in $\left(\Delta \mathrm{n}^{2}\right)$ are neglected, the equation takes the simple approximate form [compare with Eq. (6) in [2]]

$$
\nabla^{2} \mathbf{E}_{\perp}+\mathrm{k}^{2}\left(\mathrm{n}^{2} / \mathrm{n}_{0}^{2}\right) \mathbf{E}_{\perp}+2 \nabla_{\perp}\left(\mathbf{E}_{\perp} \cdot \nabla_{\perp} \Delta \mathrm{n}\right)=0
$$

If one now relies again on the SVA, Eq. (10) becomes, after writing $\mathbf{E}_{\perp}=\mathbf{A}\left(\mathbf{r}_{\perp}, \mathrm{z}\right) \exp (\mathrm{ikz})$, 


$$
\begin{aligned}
& 2 \mathrm{ik} \partial_{\mathrm{z}} \mathbf{A}+\nabla_{\perp}^{2} \mathbf{A}+2 \mathrm{k}^{2} \Delta \mathrm{n} \mathbf{A}+2 \nabla_{\perp}\left\{\mathbf{A} \cdot \nabla_{\perp}\left[\Delta \mathrm{n}+(\Delta \mathrm{n})^{2}\right]\right\} \\
& \quad=4 \nabla_{\perp}\left[(\Delta \mathrm{n})^{2} \nabla_{\perp} \cdot \mathbf{A}\right],
\end{aligned}
$$

which is, to the lowest significant order, the rigorous transverse generalization of the standard paraxial equation given by Eq. (5). The presence of the factor $(\Delta \mathrm{n})^{2}$, which can be rather small for the weakly guiding structures routinely used, appears to justify the great success the paraxial approximation has enjoyed when describing waveguide propagation. However, guiding structures with larger $(\Delta \mathrm{n})^{2}$ and smaller spatial scales are starting to play a major role in optics and require a more sophisticated description of propagation [4].

\section{CONCLUSIONS}

We have derived an exact vectorial wave quation for the transverse component of the electric field propagating in an isotropic z-independent refractive-index distribution, which, unlike the standard Helmholtz equation, does not explicitly require the use of the divergence condition. By relying on the exact Helmholtz equation, it is straightforward to derive an improved version of the standard paraxial equation.

\section{REFERENCES}

1. S. Solimeno, B. Crosignani, and P. Di Porto, Guiding, Diffraction and Confinement of Optical Radiation (Academic, 1986).

2. A. Yu. Savchencko and B. Ya. Zel'dovich, "Wave propagation in a guiding structure: one step beyond the paraxial approximation," J. Opt. Soc. Am. B 13, 273-281 (1996).

3. A. Ciattoni, P. Di Porto, B. Crosignani, and A. Yariv, "Vectorial nonparaxial propagation equation in the presence of a tensorial refractive index perturbation," J. Opt. Soc. Am. B 17, 809-819 (2000).

4. G. Brambilla, F. Xu, P. Horak, Y. Jung, F. Koizumi, N. Sessions, E. Koukharenko, X. Feng, G. Murugan, J. Wilkinson, and D. Richardson, "Optical fiber nanowires and microwires: fabrication and applications," Adv. Opt. Photon 1, 107-161 (2009). 